\title{
Space charge effects and coherent stability limits in barrier buckets
}

\author{
Oliver Boine-Frankenheim* and Ingo Hofmann \\ Gesellschaft für Schwerionenforschung, Planckstrasse 1, 64291 Darmstadt, Germany
}

(Received 27 January 2003; published 31 March 2003)

\begin{abstract}
A large-scale Vlasov simulation study of the microwave instability below transition energy in a beam confined between two barrier pulses is performed. Starting from a matched distribution function for the confined ion beam including the space charge impedance the stability threshold in the longitudinal impedance plane is obtained. A simple stability criterium is found to be in good agreement with the simulation results.
\end{abstract}

DOI: $10.1103 /$ PhysRevSTAB.6.034207

PACS numbers: 29.27.Bd, 29.20.-c, 52.59.Sa

\section{INTRODUCTION}

The determination of reliable impedance budgets for the design of low energy, high current ring machines requires the inclusion of space charge in combination with the other ring impedance components. Up to now, systematic studies of the coherent stability limits due to the longitudinal microwave instability were performed mostly for high energy bunches in conventional $\mathrm{rf}$ buckets (for a review, see, e.g., Ref. [1]). Sacherer [2] showed that, without mode coupling, a single bunch interacting with a resistive broadband impedance is stable. Nonlinear mode coupling is required in order to give an unstable, traveling wave on the bunch. However, if the instability is fast compared with the synchrotron period good agreement with growth rates from the linearized coasting beam theory is obtained. For the microwave instability in space charge dominated low energy beams detailed studies exist for coasting beams (see, e.g., [3]). The longitudinal instability in space charge dominated bunches driven by a resistive wall impedance was considered in [4-6]. Special attention was paid to the reflection of unstable space charge waves at the bunch ends. Experiments on the reflection of space charge waves were described in Ref. [7].

In the present work a large-scale simulation study of the microwave instability in a barrier bucket below transition energy is performed. In an ideal barrier bucket the bunch is confined between two well-separated and localized rf pulses. Between the pulses the beam is coasting. This enables a straightforward comparison of bunched and coasting beam stability limits. The effect of the bunch is more or less reduced to a gap in the coasting beam. However, the results obtained in this work are also applicable to general bunch forms.

Barrier buckets are usually considered in low energy, high current ring machines in order to increase the transverse space charge limit [8]. The flattening of the bunch shape, compared with bunches in conventional rf buckets,

*Electronic address: O.Boine-Frankenheim@gsi.de causes a reduction of the peak current density and thus of the transverse space charge tune shift. Another method to flatten the bunches is the installation of a secondharmonic rf system [9]. The bunching factor, determined by the average current divided by the peak current, can be as high as 0.5 in these $\mathrm{rf}$ systems. The highest bunching factors, approaching one, can be achieved with barrier buckets. Therefore, barrier buckets play an important role in proposed accumulators for low to medium energy, high current ion beams, like the planed SIS 100 synchrotron at GSI [10] or the storage rings for heavy ion fusion driver scenarios [11]. Experiments with medium energy proton beams in barrier buckets were performed already in the BNL Alternating Gradient Synchrotron (AGS) [8] and in the CERN Super Proton Synchrotron (SPS) [12]. In the AGS experiment the two barriers were formed by a single sine wave that was generated by a magnetic alloy loaded cavity. The comparison of the AGS experiment with simulations including the shunt impedance of the barrier rf cavity showed the importance of a feedback system. The effect of parametric resonances in barrier buckets excited by rf noise was studied theoretically in Ref. [13]. Besides the increased transverse space charge limit barrier buckets offer greater flexibility for bunch manipulations compared to conventional rf systems, especially for injection and accumulation (see, e.g., [14]). Recently the application of barrier buckets to future high energy colliders was proposed [15]. Higher luminosities could be achieved because of the reduced beam-beam tune shift. Another application is the cooling of bunched ion beams by electrons or lasers. In Refs. $[16,17]$ it was demonstrated experimentally that laser cooling in a barrier bucket generates extremely cold, space charge dominated ion bunches.

The paper is structured as follows. Section II describes the framework of the kinetic model. In Sec. III a matched beam distribution with space charge is introduced that serves as the initial distribution in the simulations. Section IV then outlines the linearized theory for bunched beams before in Sec. V the simulation results are presented and analyzed. Some design considerations for barrier bucket systems are given in Sec. VI. 


\section{KINETIC DESCRIPTION}

The time evolution of the beam distribution $f\left(z, v_{z}, t\right)$ is governed by the Vlasov equation

$$
\frac{\partial f}{\partial t}+v_{z} \frac{\partial f}{\partial z}-\frac{q \eta}{\gamma_{0} m} E_{z} \frac{\partial f}{\partial v_{z}}=0
$$

with the distance $z$ and the velocity derivation $v_{z}$ from the synchronous particle, the total longitudinal electric field $E_{z}(z, t)$, the relativistic parameter $\gamma_{0}=\left(1-\beta_{0}^{2}\right)^{1 / 2}$, the slip factor $\eta$, the ion charge $q$, and the ion mass $m$. The line charge density $\rho_{L}$ is given through

$$
\rho_{L}(z, t)=q \int_{-\infty}^{\infty} f d v_{z}
$$

The electric field amplitude resulting from a line density perturbation at the $n$th harmonic of the revolution frequency $\omega_{0}$ is obtained from

$$
E_{n}=-\frac{1}{2 \pi R} Z_{n} I_{n}
$$

Here, $I_{n}=\beta_{0} c \rho_{L, n}$ and $E_{n}$ denote the amplitudes of the beam current and the resulting longitudinal electric field. $Z_{n}$ is the total ring impedance acting at the $n$th harmonic. At low beam energies the space charge impedance is usually dominant. For the case of a transversely uniform beam of radius $a$ in a circular beam pipe of radius $b$ it was shown in Refs. $[18,19]$ that the longitudinal space charge impedance can be approximated as

$$
\frac{Z_{n}^{\mathrm{sc}}}{n}=-i \frac{g Z_{0}}{\beta_{0} \gamma_{0}^{2}} \frac{1}{1+\left(n / n_{c}\right)^{2}},
$$

with $Z_{0}=377 \Omega, g=0.5+2 \ln (b / a)$, and the cutoff harmonic $n_{c}=2 \pi R / \lambda_{c}$ determined by the length scale parameter $\lambda_{c}=\gamma_{0}^{-1} \pi a \sqrt{g}$. The space charge impedance is proportional to the harmonic number in the long wavelength regime and vanishes at wavelengths of the order of $\lambda_{c}$ due to the image charges on the inner side of the beam pipe.

In addition to the purely reactive space charge impedance [Eq. (4)] many of the vacuum chamber elements through which the beam travels have a cavitylike structure whose individual contributions to the total ring impedance can be represented as (shunt impedance $R_{s}$, quality factor $Q$ )

$$
Z_{n}^{\mathrm{cav}}=\frac{R_{s}}{1+i Q\left(\omega_{n} / \omega_{r}-\omega_{r} / \omega_{n}\right)},
$$

with the eigenfrequency $\omega_{r}$ and $\omega_{n}=n \omega_{0}$. Often the total impedance due to imperfections of the ring environment is approximated in terms of a single broadband resonator impedance with $\omega_{r}$ in the $\mathrm{GHz}$ range.

\section{MATCHED BEAM DISTRIBUTIONS WITH SPACE CHARGE}

\section{A. Parabolic bunch model}

For long bunches $\left(l \gg \lambda_{c}\right)$ in the presence of space charge, Neuffer (Ref. [20]) showed that the distribution function

$$
f(z, v)=f_{0} \sqrt{1-\frac{z^{2}}{z_{m}^{2}}-\frac{v^{2}}{v_{m}^{2}},} \quad-z_{m}<z<z_{m},
$$

with the bunch half length $z_{m}=l / 2$, the velocity halfwidth $v_{m}$, and

$$
f_{0}=\frac{3 N}{2 \pi v_{m} z_{m}}
$$

reproduces the line charge density (particle number $N$ )

$$
\rho_{L}(z)=\rho_{m}\left(1-\frac{z^{2}}{z_{m}^{2}}\right), \quad \rho_{m}=q \frac{3 N}{4 z_{m}}
$$

that yields a linear space charge electric field. In the case of an external linear ("sawtooth") electric field or in a sinusoidal $\mathrm{rf}$ field for small particle amplitudes $\left(z_{m} \ll\right.$ $\lambda_{0} / 2, \mathrm{rf}$ wave length $\lambda_{0}$ ) the Neuffer distribution (also called the parabolic bunch model) represents a selfconsistent solution of the Vlasov equation [Eq. (1)] in the presence of space charge [Eq. (4)]. It is important to point out that a matched distribution in an arbitrary external rf field can be obtained using a technique described in Ref. [21].

Within the parabolic bunch model the matched envelope can be calculated from (see also Ref. [22])

$$
k_{0}^{2} z_{m}-\frac{K_{L}}{z_{m}^{2}}-\frac{\epsilon_{L}^{2}}{z_{m}^{3}}=0,
$$

with $k_{0}^{2}=q V_{0} h \eta /\left(2 \pi R^{2} \gamma_{0} \beta_{0}^{2} m c^{2}\right)$ the linearized $\mathrm{rf}$ focusing force for a voltage amplitude $V_{0}$ at harmonic $h$, the longitudinal perveance

$$
K_{L}=-\frac{3 g N r_{c}}{2 \beta_{0}^{2} \gamma_{0}^{3}} \eta,
$$

with the classical particle radius $r_{c}, q$ as the charge, and $m$ the ion mass. The longitudinal emittance is defined as

$$
\epsilon_{L}=\frac{v_{m} z_{m}}{\beta_{0} c}=-\eta z_{m} \Delta_{m}
$$

with the momentum spread $\Delta_{m}$. The perveance can be related to the bunched beam space charge parameter (see Ref. [23])

$$
\begin{aligned}
& \Sigma=\frac{2 K_{L} z_{m}}{\epsilon_{L}^{2}}=\frac{q g I_{m}}{\pi \epsilon_{0} m\left(c \beta_{0} \gamma_{0}\right)^{3}|\eta| \Delta_{m}^{2}}, \\
& I_{m}=q \beta_{0} c \rho_{m} .
\end{aligned}
$$

The required rf voltage amplitude for a given $z_{m}$ can be calculated from 


$$
k_{0}^{2}=\frac{|\eta| \Delta_{m}^{2}}{z_{m}^{2}}\left(1+\frac{\Sigma}{2}\right)
$$

The depression of the synchrotron wave number due to space charge is given through

$$
\frac{k}{k_{0}}=\left(1+\frac{\Sigma}{2}\right)^{-1 / 2} \text {. }
$$

From this we can obtain the reduced momentum acceptance $\Delta_{\max }$ due to space charge in a sinusoidal rf bucket

$$
\Delta_{\max }^{2}=\frac{k^{2}}{k_{0}^{2}} \Delta_{\max , 0}^{2}
$$

with the momentum acceptance for $\Sigma=0$

$$
\Delta_{\text {max }, 0}^{2}=\frac{2 q V_{0}}{\pi h|\eta| \beta_{0} c p_{0}} .
$$

\section{B. "Sausage" beam model}

By extending the parabolic bunch model to a beam confined between two sinus half waves ("sausage beam model") we arrive at the distribution function (see Fig. 1)

$f(z, v)= \begin{cases}f_{0} \sqrt{1-\frac{(z+l / 2)^{2}}{z_{m}^{2}}-\frac{v^{2}}{v_{m}^{2}}}:-l / 2-z_{m}<z<-l / 2, \\ f_{0} \sqrt{1-\frac{v^{2}}{v_{m}^{2}}} \quad:-l / 2<z<l / 2, \\ f_{0} \sqrt{1-\frac{(z-l / 2)^{2}}{z_{m}^{2}}-\frac{v^{2}}{v_{m}^{2}}}: l / 2<z<l / 2+z_{m},\end{cases}$

with

$$
f_{0}=\frac{\rho_{m}}{q \pi v_{m}} .
$$

The resulting line charge density is

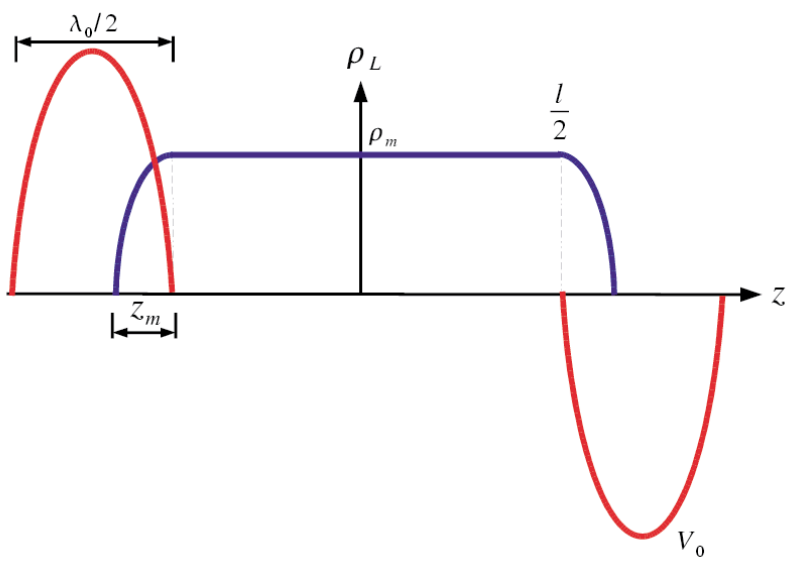

FIG. 1. (Color) Sketch of the line density (blue curve) of a matched beam confined between two sinus half waves (red curve) of amplitude $V_{0}$.

$$
\rho_{L}(z)= \begin{cases}\rho_{m}\left(1-\frac{(z+l / 2)^{2}}{z_{m}^{2}}\right) & :-l / 2-z_{m}<z<-l / 2, \\ \rho_{m} & :-l / 2<z<l / 2 \\ \rho_{m}\left(1-\frac{(z-l / 2)^{2}}{z_{m}^{2}}\right) & : l / 2<z<l / 2+z_{m}\end{cases}
$$

with

$$
\rho_{m}=\frac{q N}{l+\frac{4}{3} z_{m}} .
$$

The sausage beam model represents a self-consistent solution for a beam confined between two triangular pulses. It can be applied to sinus waves, provided that the penetration depth $z_{m}$ is much smaller than $\lambda_{0} / 2$. As for the parabolic bunch we can use the normalized space charge parameter [Eq. (12)] together with Eq. (13) to calculate the required $\mathrm{rf}$ amplitude for a given penetration depth $z_{m}$.

The synchrotron period $T_{s}$ in a barrier bucket is determined by the time of flight between the two barriers plus the time spent in the barriers that can be neglected because of $\lambda_{0} \ll l$ and $\beta c \gg v_{m}$,

$$
T_{s}=\frac{2 l}{v}+\frac{\lambda_{0}}{\beta c}\left(1+\frac{\Sigma}{2}\right)^{1 / 2} \approx \frac{2 l}{v} .
$$

\section{STABILITY THRESHOLDS FOR THE MICROWAVE INSTABILITY FROM LINEARIZED THEORY}

Stability thresholds for the coasting beam microwave instability are usually given in the normalized impedance plane $(U, V)$ (see, e.g., Ref. [24])

$$
V_{n}-i U_{n}=\frac{2 I_{0} q}{\pi m c^{2} \beta_{0}^{2} \gamma_{0}|\eta| \Delta_{\text {FWHM }}^{2}}\left[\operatorname{Re}\left(\frac{Z_{n}}{n}\right)+i \operatorname{Im}\left(\frac{Z_{n}}{n}\right)\right],
$$

with the average current $I_{0}$, the ring impedance $Z_{n}$, and the full width half maximum (FWHM) momentum spread $\Delta_{\text {FWHM }}$.

For a general operating point in $\left(U_{n}, V_{n}\right)$ space one needs to evaluate the dispersion function for the complex frequency shift $\Delta \omega=\omega-n \omega_{0}$ following from the linearized Vlasov equation. This determines the corresponding region of stability $\left(\Delta \omega_{I} \leq 0\right)$ that is provided by Landau damping.

Below transition and in the cold beam approximation $\left(U_{n}^{2}+V_{n}^{2} \gg 1\right)$ a simplified dispersion function can be obtained (Ref. [24]) yielding backward ("slow") and forward ("fast") running waves with a coherent phase velocity $\left(v_{\mathrm{FWHM}}=-\eta \beta_{0} c \Delta_{\mathrm{FWHM}}\right)$

$$
c_{s}= \pm \frac{1}{2} v_{\mathrm{FWHM}}\left(\frac{1}{2} \sqrt{U_{n}^{2}+V_{n}^{2}}+\frac{1}{2} U_{n}\right)^{1 / 2} \text {. }
$$

The instability growth (damping) rate for the slow (fast) 
wave is

$$
\tau_{I}^{-1}=\frac{1}{2} \frac{n}{R} v_{\text {FWHM }}\left(\frac{1}{2} \sqrt{U_{n}^{2}+V_{n}^{2}}-\frac{1}{2} U_{n}\right)^{1 / 2} .
$$

In the frame comoving with the synchronous particle the instability is convective, meaning the unstable mode is a traveling wave. On the contrary the pure $\left(V_{n}=0\right)$ negative mass instability above transition is absolute (the unstable mode is a standing wave). It is usually assumed that the coasting beam results for the complex frequency shift can be applied to bunched beams provided that the bunch length $l$ is much longer than the wavelength $\lambda_{n}=$ $2 \pi n / R$ of interest. The results presented in a later section will show that this is not the case in space charge dominated beams below transition.

In low energy beams the imaginary part of the impedance spectrum $U_{n}$ is dominated by the space charge impedance [Eq. (4)]. The normalized space charge impedance $U$ for $n \ll n_{c}$ can be related to the bunched beam space charge parameter $\Sigma$ through

$$
U=\frac{1}{\delta^{2}} \Sigma
$$

with $I_{0}=I_{m}$ and the constant parameter $\delta$ depending on the relation between the momentum spreads $\Delta_{\text {FWHM }}=$ $\delta \Delta_{m}$. For the parabolic and the sausage bunch models $\delta=\sqrt{3}$ holds. Coasting beams are called space charge dominated for $U \gtrsim 1$. Below transition the convection induced by the space charge impedance has strong implications on the stability of bunched beams, as will be discussed in the following.

Within a linearized theory a single long bunch interacting with a broadband resonator is always stable: perturbations generated at one of the bunch ends move towards the other end where they are reflected. As predicted in Ref. [4] already, due to the fact that the backward running (slow) mode growths and the forward running (fast) mode is damped the total growth over one round trip vanishes. However, as pointed out in Ref. [6], unstable growth over one round trip is possible due to mode conversion during reflection of finite amplitude perturbations. On the other hand, as pointed out in Ref. [5], mode conversion and wave-particle coupling during reflection also have a self-limiting effect on the instability. In the framework of the cold coasting beam approximation both pictures can be cast into a simple "stability criterion" for long bunches

$$
\left(\alpha_{e} \tau_{I}\right)^{-1} \lesssim \frac{c_{s}}{l} .
$$

Here $\alpha_{e}$ is introduced as the allowed end-to-end $e$-folding before nonlinear effects at the boundary lead to unstable growth or as the damping rate $\alpha_{e} c_{s} / l$ due to wave reflection. Using Eqs. (23) and (24) yields

$$
\frac{V_{n}}{U_{n}} \lesssim\left[\left(\frac{1+\left[\alpha_{e} R /(n l)\right]^{2}}{1-\left[\alpha_{e} R /(n l)\right]^{2}}\right)^{2}-1\right]^{2} .
$$

For $\alpha_{e} R /(n l) \ll 1$ Eq. (27) can be approximated through

$$
\frac{V_{n}}{U_{n}} \lesssim \frac{2 R \alpha_{e}}{n l}
$$

The stability criteria [Eqs. (26) and (28)] predict that space charge induced convection can have a stabilizing effect on the microwave instability in bunches. The factor $\alpha_{e}$ must be determined from simulation studies described in the following section.

It is worth keeping in mind that in the limiting case of very long bunches $(l \rightarrow \infty)$ the damping effect due to wave reflection gets weaker and coasting beam damping rates such as the Landau damping rate $\tau_{L}^{-1}(n)$ due to Gaussian momentum spread tails (see Ref. [3]) determine the instability threshold. Therefore Eqs. (27) or (28) can be valid for $\alpha_{e} c_{s} / l \gg \tau_{L}^{-1}(n)$ only.

\section{SIMULATION OF THE MICROWAVE INSTABILITY IN BARRIER BUCKETS}

The microwave instability in a single bunch is essentially a nonlinear phenomenon. Therefore the reliable determination of threshold currents or impedance budgets requires analytic or numerical methods beyond linearized Vlasov theory. In the present work a large scale simulation study in $\left(U_{n}, V_{n}\right)$ parameter space ("impedance scan") is performed employing a Vlasov code (Ref. [3]). Especially for resolving instability thresholds a high-resolution, "noise-free" Vlasov solver has advantages over standard particle tracking codes. In the simulations we study the interaction of the beam with a cavity impedance in the presence of space charge. For the space charge impedance we choose a cutoff parameter $\lambda_{c}=$ $0.1 \mathrm{~m}$. In order to limit the number of points along the $z$ axis to $N_{z}=1024$ with $\Delta x \ll \lambda_{c}$ we restrict our numerical study to a periodic beam pipe of the length $L=$ $10 \mathrm{~m}$. The resonance of the resonator is tuned to the harmonic number $n_{r}=20$ of the simulation model and the quality factor is set to $Q=5$. A two parameter study is performed by varying the shunt impedance $R_{s}$ of the resonator and the space charge reactance $X_{\mathrm{sc}}=$ $g Z_{0} /\left(\beta_{0} \gamma_{0}^{2}\right)$. An initial harmonic perturbation at $n=n_{r}$ with a maximum amplitude of $1 \%$ is used to initiate the instability.

\section{A. Coasting beam impedance scans}

In the limiting case of a coasting beam $(l \rightarrow \infty)$ with a Gaussian velocity distribution the momentum spread blowup from about 100 Vlasov simulation runs for different $(U, V)$ is shown in Fig. 2. Here $U$ and $V$ are the normalized space charge and resonator shunt impedance, respectively. We see that the boundary of the stable (black) area in $(U, V)$ space agrees very well with the stability threshold obtained from the linearized Vlasov theory for a Gaussian velocity distribution (white line). For the coasting beam velocity distribution, 


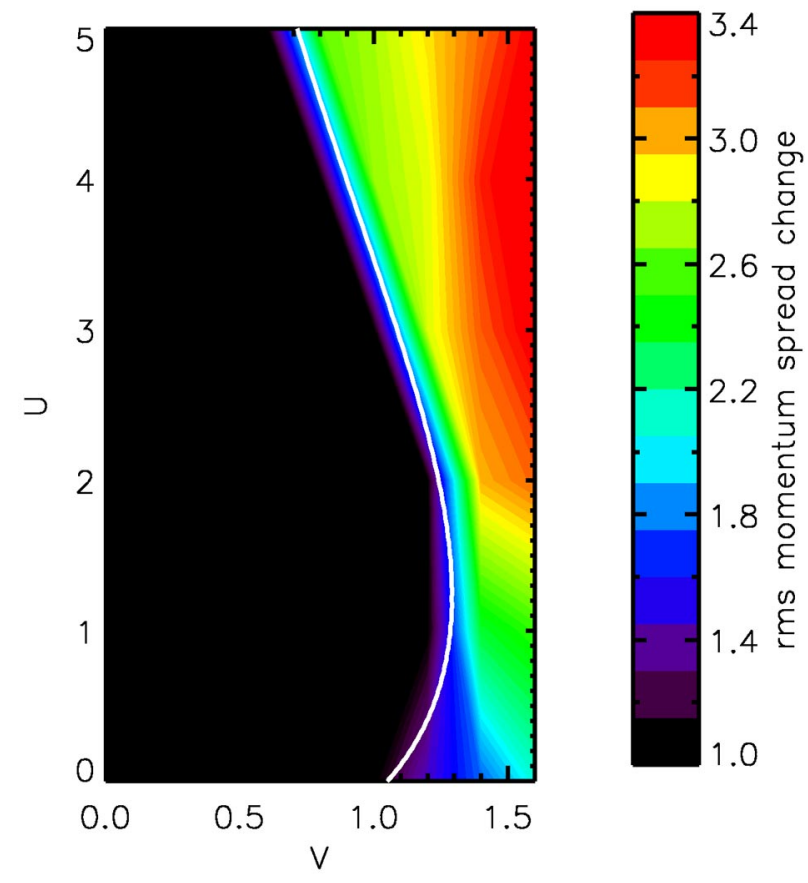

FIG. 2. (Color) Momentum spread increase in a coasting beam with a Gaussian velocity distribution as a function of initial $(U, V)$ values. The black area corresponds to a stable beam.

$$
f(v)=f_{0} \sqrt{1-\frac{v^{2}}{v_{m}^{2}}}
$$

that directly corresponds to a sausage beam [Eq. (17)] with $l \rightarrow \infty$, the result is shown in Fig. 3. The stability

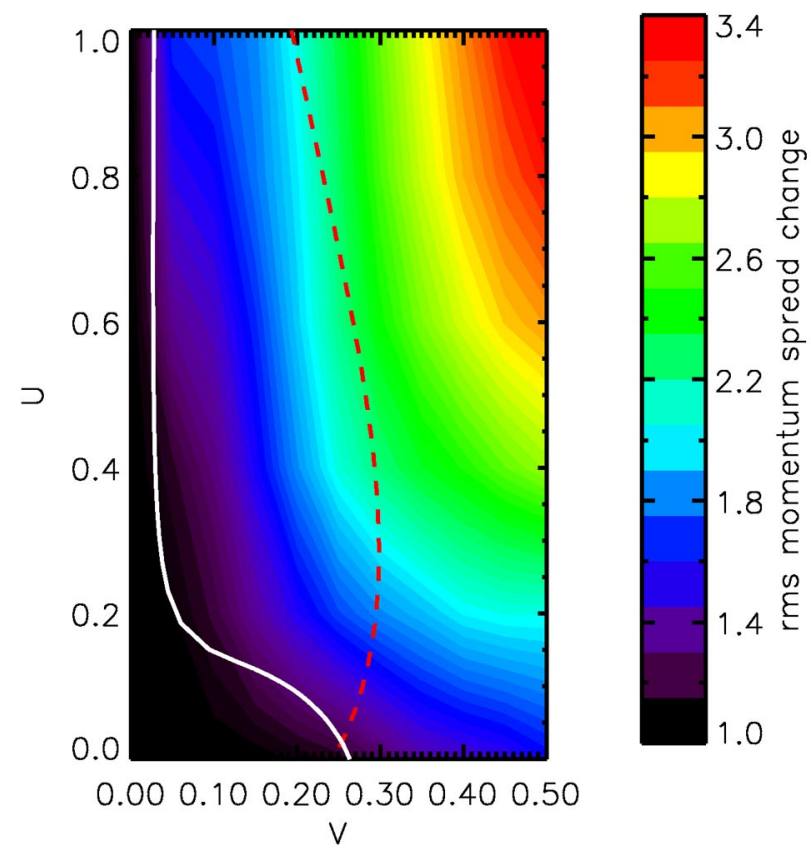

FIG. 3. (Color) Momentum spread increase in a coasting beam with the velocity distribution Eq. (29) as a function of initial $(U, V)$ values. The black area corresponds to a stable beam. threshold obtained from linearized Vlasov theory (solid white line) agrees with the simulation result if a damping rate $\nu \approx 10 \mathrm{~s}^{-1}$ is added to the denominator of the dispersion integral. This damping rate slightly enlarges the stable area and removes the singular dip in the linearized stability boundary for $V=0$ that cannot be resolved within the simulation. This damping rate reflects the unavoidable numerical diffusion in grid based simulation codes. The comparison with the instability threshold curve for the rms equivalent $\left[v_{m}=2 v_{\text {rms }}\right.$ for Eq. (29)] Gaussian velocity distribution (dashed red line in Fig. 3) shows that the stable area is reduced, but the rms momentum spread growth inside the area between the two curves remains below 2.0.

\section{B. Barrier bucket impedance scans}

In the bunched beam computer model an initial sausage beam with $l \approx 7 \mathrm{~m}$ and $z_{m} \approx 1 \mathrm{~m}$ is studied. The bunch is confined between two triangular shaped electric field pulses of different polarity (the total pulse width corresponds to the third harmonic of the simulation model). The electric field gradient is matched to the initial beam conditions using Eq. (13). Figure 4 shows the bunch with a superimposed modulation at $n=20$ with an amplitude variation that is typically generated by the interaction with a cavity impedance. The current amplitudes of the modulated bunch together with the resistive part of the cavity impedance and the space charge impedance are shown in Fig. 5. In order to avoid overlap of the resistive cavity impedance with the unperturbed bunch spectrum the resonance harmonic $n_{r}$ and the quality factor $Q$ are chosen sufficiently large. Still the amplitude spectrum of the unstable mode is narrow compared to the chosen frequency width of the resistive resonator impedance.

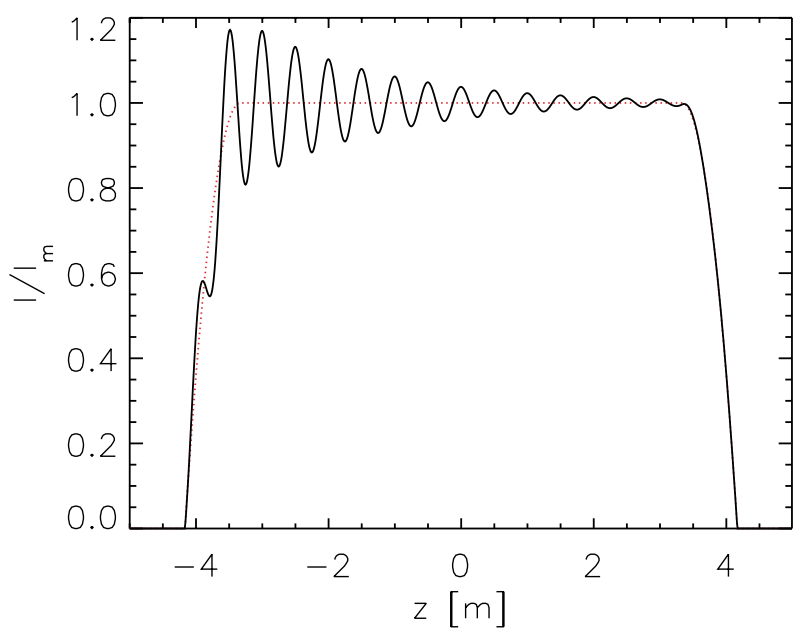

FIG. 4. (Color) Typical standing wave pattern on a sausage beam induced by a resonator impedance. The red dotted curve represents the unperturbed current profile. 


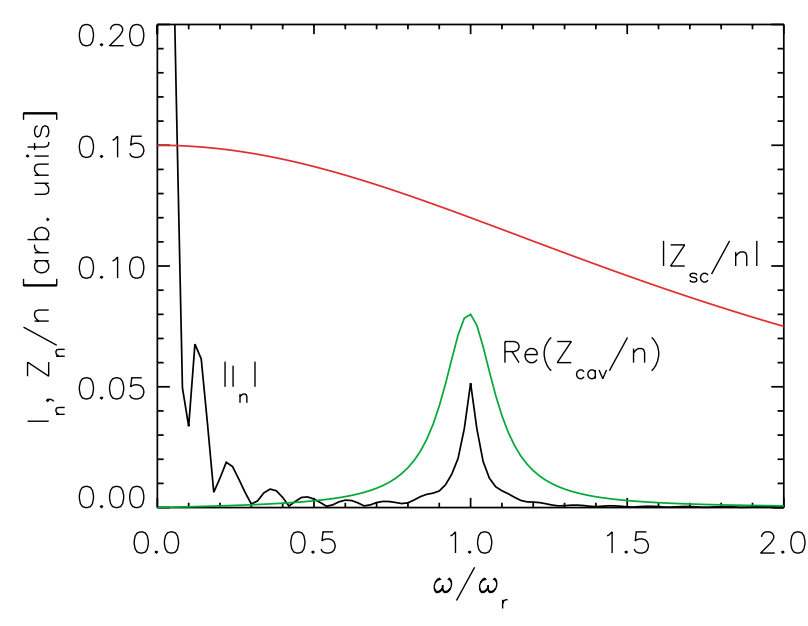

FIG. 5. (Color) Current amplitude of the modulated bunch (Fig. 4) as a function of the frequency together with the resistive part of the cavity impedance and the space charge impedance (both in a.u.).

The result of a parameter scan in $(U, V)$ space for a cavity impedance centered at $n_{r}=20$ is shown in Fig. 6 . For $U \lesssim 1$ the stable area increases relative to the equivalent coasting beam (solid white line) and extends towards the stability domain for the rms equivalent coasting beam with a Gaussian velocity distribution (dashed red line). For $U \geqslant 1$ the stable area increases relative to a coasting beam and the instability threshold can be well described (dashed white line) by Eq. (27) or its approximation Eq. (28) with $n=n_{r}=20$ and an endto-end $e$-folding factor $\alpha_{e} \approx 6$. The dependence on the

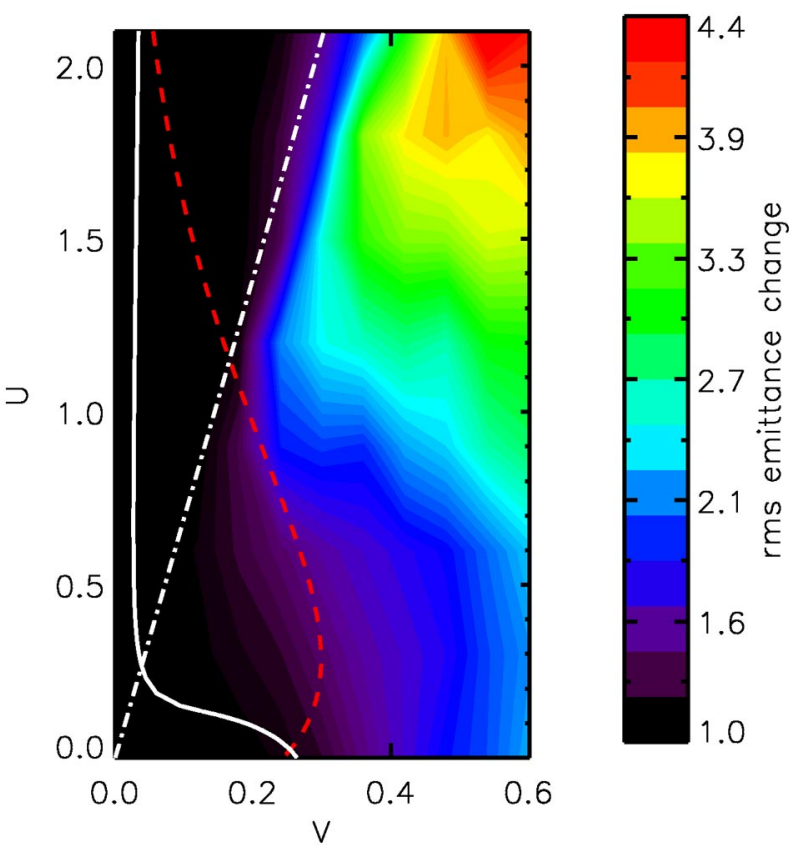

FIG. 6. (Color) Emittance increase in a sausage beam as a function of initial $(U, V)$ values with $n_{r}=20$. The black area corresponds to a stable beam. $\alpha_{e}=6$.

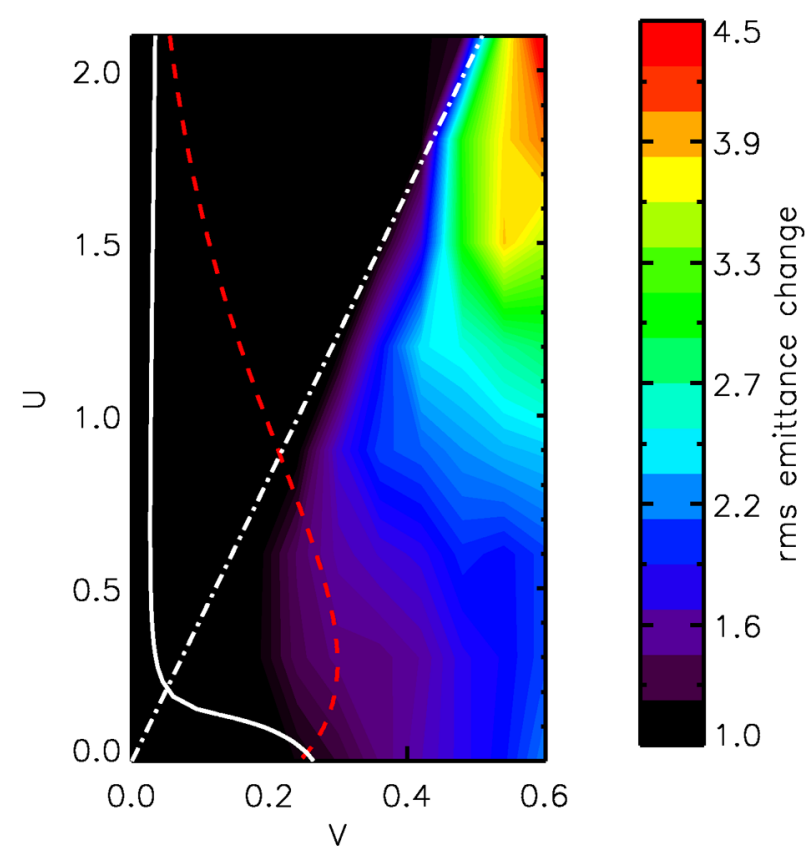

FIG. 7. (Color) Emittance increase in a sausage beam as a function of initial $(U, V)$ values with $n_{r}=10$. The black area corresponds to a stable beam. $\alpha_{e}=5$.

initial perturbation amplitude was checked between 0.1 and $1 \%$. In this range the boundary of the black area stays unaffected.

The results for decreased resonance harmonics $n_{r}=10$ and $n_{r}=5$ are shown in Figs. 7 and 8. For $n_{r}=10$ the

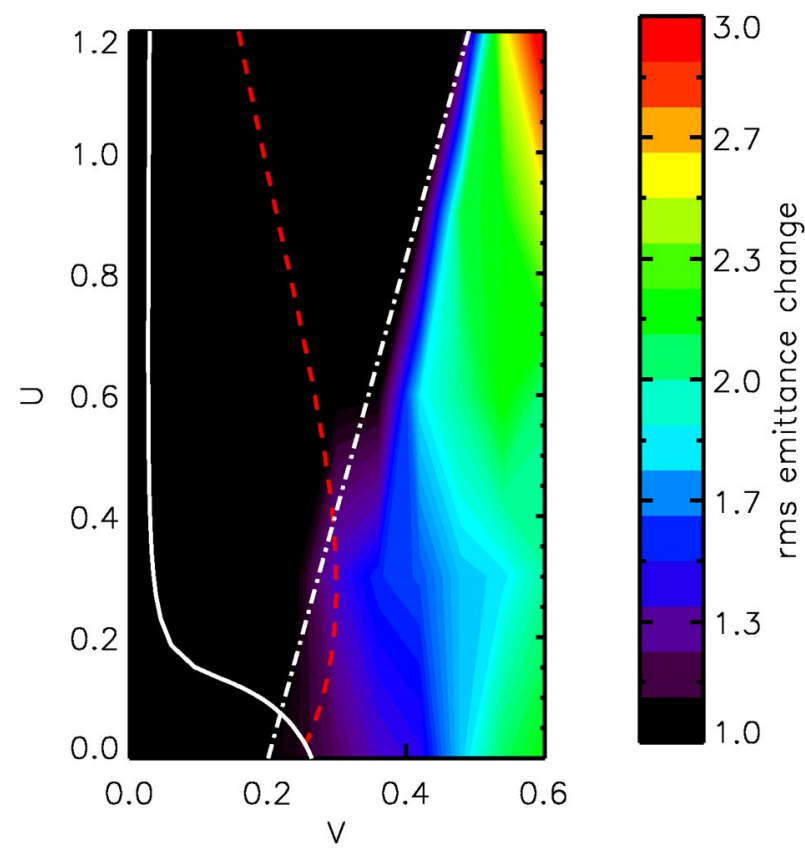

FIG. 8. (Color) Emittance increase in a sausage beam as a function of initial $(U, V)$ values with $n_{r}=5$. The black area corresponds to a stable beam. $\alpha_{e}=3$. 
stability threshold can again be well described by Eq. (27) with a slightly decreased $\alpha_{e} \approx 5$. For $n_{r}=5$ the wavelength of the unstable mode is close to the pulse width of the barrier and in addition the bunch cannot be regarded as long relative to the unstable wavelength. Still the stable area for $U \gtrsim 1$ can be fitted to Eq. (27) if the origin is shifted and $\alpha_{e} \approx 3$ is chosen.

\section{Instability saturation in barrier buckets}

By studying the time evolution towards a new stationary state for initial parameters close to the stability boundaries one can gain insight into the relevant damping mechanisms for finite amplitude modes in barrier buckets. In addition one can learn how far outside the stable (black) areas in Figs. 6-8, a machine can be operated safely. Figure 9 shows the evolution of the instability for initial parameters $U=0.3, V=0.3$, and
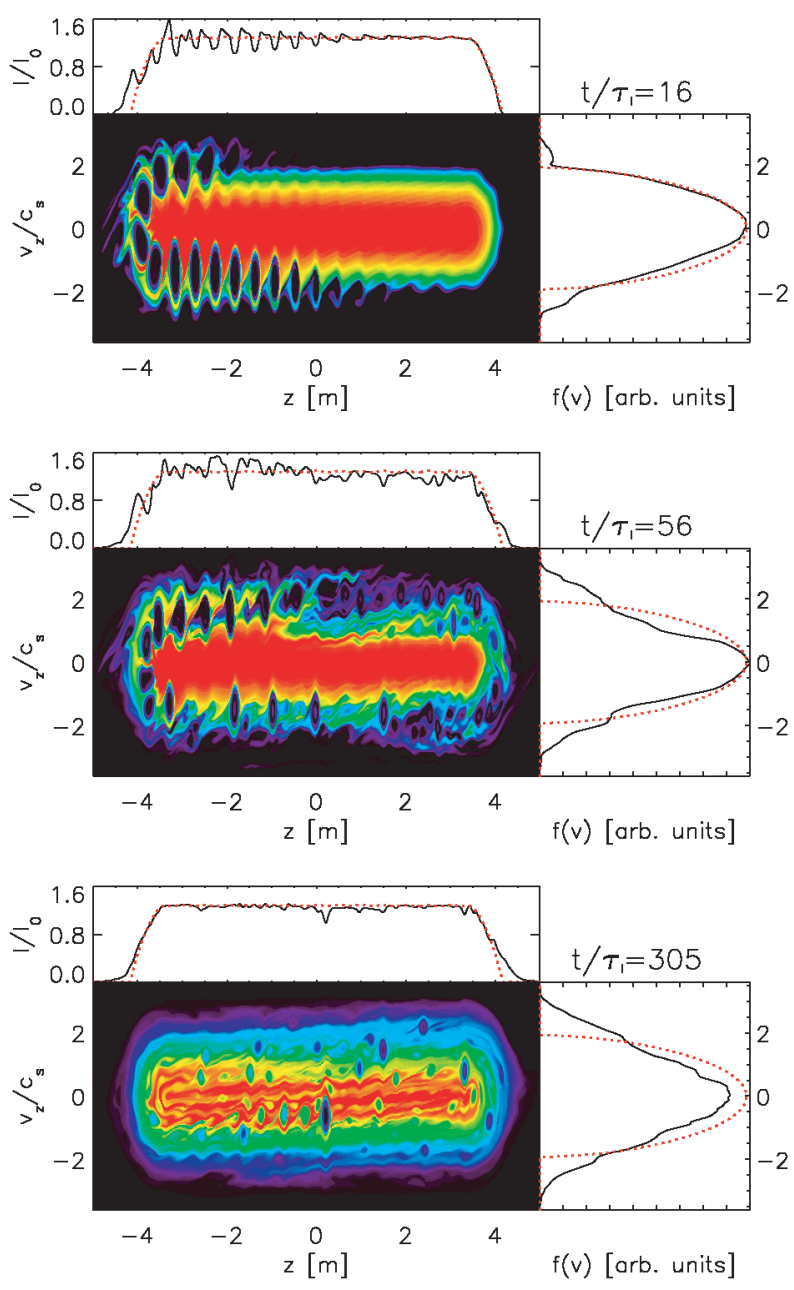

FIG. 9. (Color) Time evolution of the microwave instability in a barrier bucket for $U=0.3, V=0.3$, and $n_{r}=20$. The red dotted curves represent the initial current profile and velocity distribution. $n_{r}=20$ (final $\mathrm{rms}$ emittance change approximately 1.6). The cold beam growth time $\tau_{I}$ [Eq. (24)] and the coherent phase velocity $c_{s}$ [Eq. (23)] are used to normalize time and velocity. One has to keep in mind that these cold beam values are not applicable for initial values $U^{2}+$ $V^{2}<1$. However, in the present example they are used also to normalize velocity and time in the case of a "warm" beam. In Fig. 9 one can see that the slow mode is amplified towards its way to the bunch end. Before reaching the bunch end particles are trapped in the large amplitude wave. The phase space holes formed by the trapped particles survive the reflection, but the amplitude of the reflected mode is strongly reduced, possibly due to nonlinear wave phenomena during reflection, such as mode coupling and wave-particle interaction. After more than $300 \times \tau_{I}$ one can still observe long-lived hole structures in the turbulent phase space distribution. The velocity distribution turns into a stationary tentlike
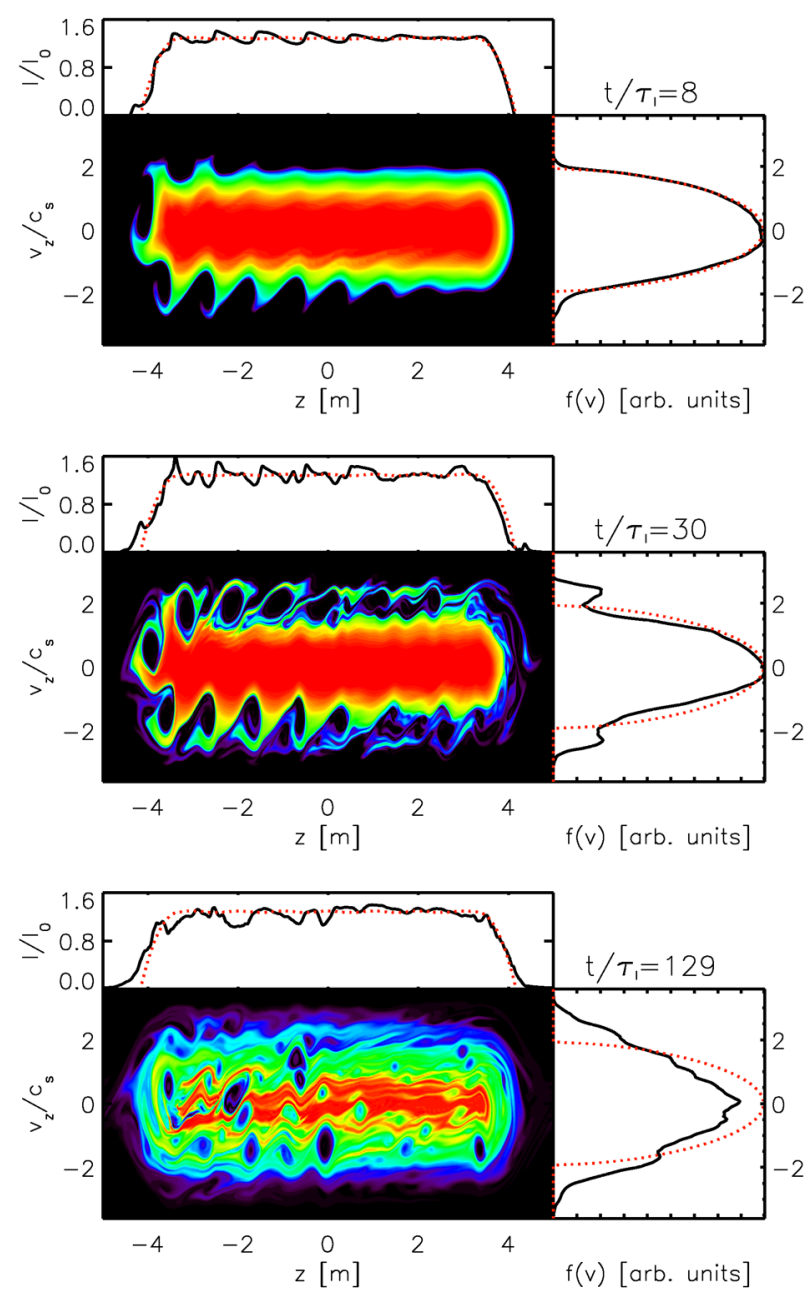

FIG. 10. (Color) Time evolution of the microwave instability in a barrier bucket for $U=0.3, V=0.3$, and $n_{r}=10$. The red dotted curves represent the initial current profile and velocity distribution. 
form, with an increased total width relative to the initial distribution. No particle leakage into the gap is observed. The time evolution for $U=0.3, V=0.3$, and $n_{r}=10$ in Fig. 10 shows a similar behavior. For $U \leq 1$ one can safely operate inside the area enclosed by the stability curve for the coasting rms equivalent Gaussian beam, if an rms emittance change of 1.6 with a corresponding increased penetration depth into the gap of about $30 \%$ is acceptable.

In the space charge dominated regime a different saturation behavior is observed. The time evolution for $U=$ $1.0, V=0.2$, and $n_{r}=20$ is shown in Fig. 11. Here the final rms emittance change is 1.5 , similar to the former examples, but the shape of the final distribution function is very different. Particles trapped and accelerated in the large amplitude wave form a low density halo. The cor-
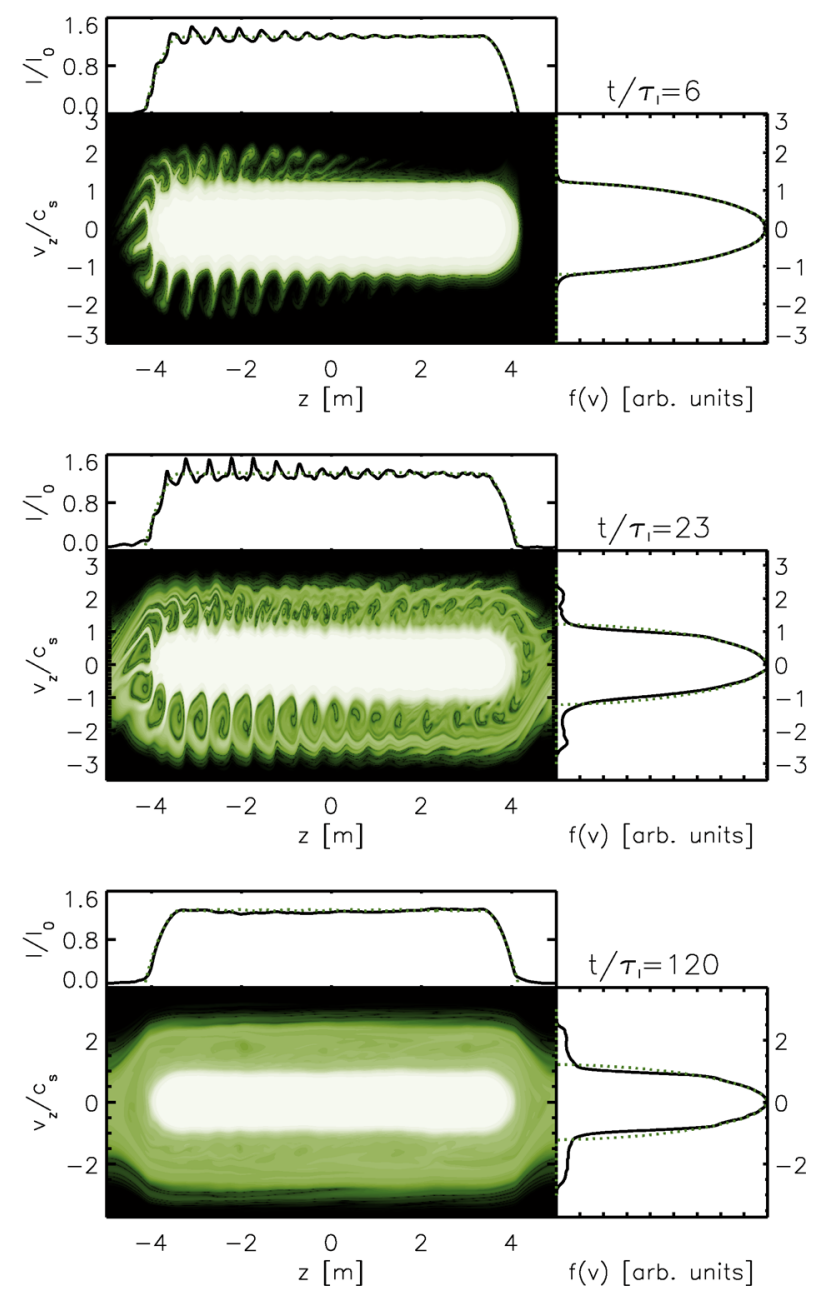

FIG. 11. (Color) Time evolution of the microwave instability in a barrier bucket for $U=1.0, V=0.2$, and $n_{r}=20$. For a better resolution of the low density phase space regions the contours of $\ln (1+f)$ are shown and a different color set is used. The green dotted curves represent the initial current profile and velocity distribution.
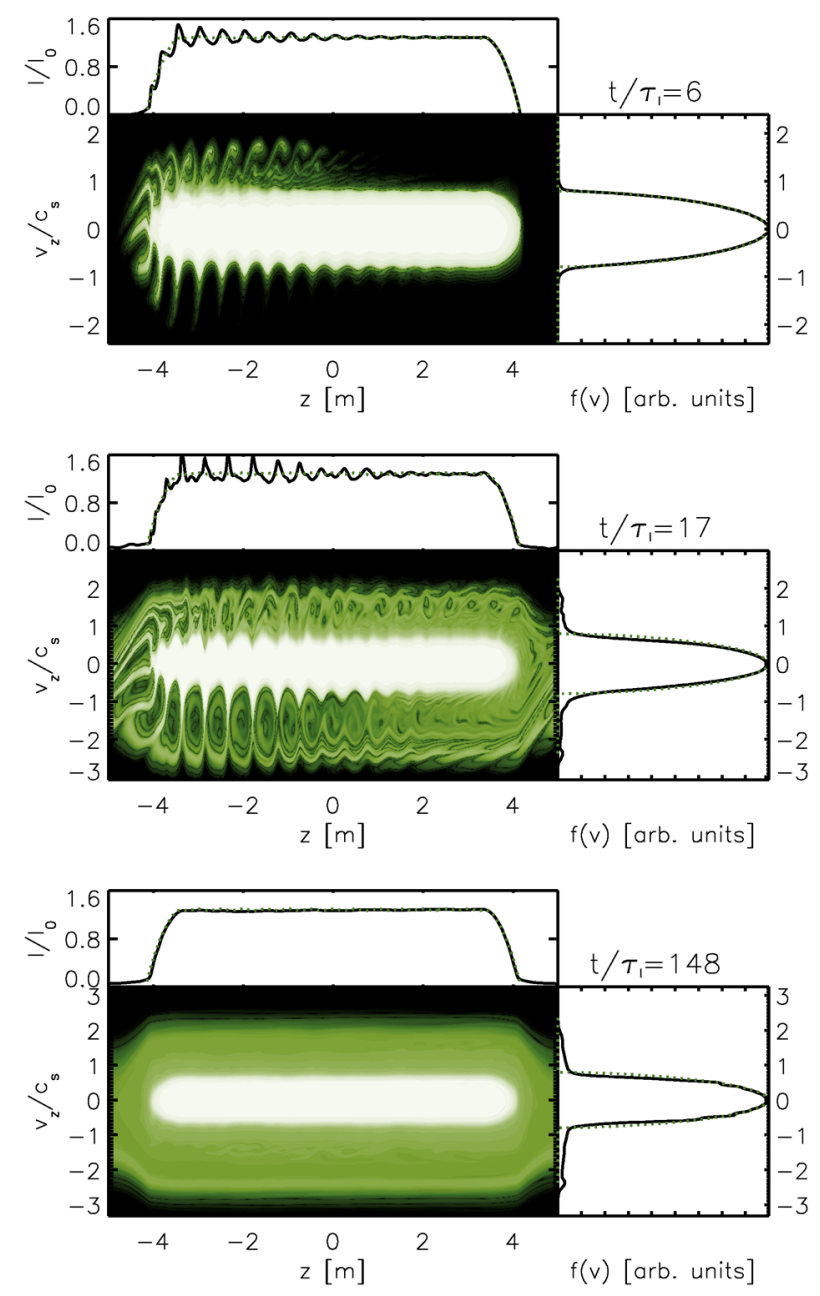

FIG. 12. (Color) Time evolution of the microwave instability in a barrier bucket for $U=2.0, V=0.3$, and $n_{r}=20$. For a better resolution of the low density phase space regions the contours of $\ln (1+f)$ are shown and a different color set is used. The green dotted curves represent the initial current profile and velocity distribution.

responding tail of the velocity distribution extends up to $v \approx 2 c_{s}$. This can be related to the fact that large amplitude perturbations move faster then $c_{s}$ (see, e.g., [3]). For the chosen barrier height, some of the tail particles can leak into the gap. The tail efficiently seems to damp all current perturbations on the bunch. The simulations show that this saturation mechanism is characteristic for operating points with $U \gtrsim 1$ outside, but still close to the stable area.

For increasing $U$ the tails get thinner and extend to higher maximum velocities. Figure 12 shows the time evolution for $U=2.0, V=0.3$, and $n_{r}=20$. Here the tail extends up to $2.5 c_{s}$ for the slowed down (negative $v_{z}$ ) particles. For $U \gtrsim 1$ one must be careful in choosing operating points outside the black areas in Figs. 6-8 because of the possible penetration of particles slowed down by large amplitude waves into the gap. 


\section{BARRIER BUCKET DESIGN CONSIDERATIONS}

\section{A. Barrier height considerations}

The required barrier height depends on the tolerable penetration depth of particles into the gap. If, e.g., the gap size is adjusted to the extraction kicker rise time, then particles between the barrier pulses are lost. In high current machines this may lead to residual activation. Beam ions in the gap can also cause a two-stream instability between beam ions and residual gas electrons (Ref. [25]). For the suppression of this two-stream instability in long bunches clean gaps are essential.

For the sausage beam distribution, or rms equivalent distribution functions, the penetration depth $z_{m}$ for a given voltage can be obtained from Eq. (13). For space charge dominated beams $(U \gtrsim 1)$ and $V=0$ the required barrier voltage scales according to $V_{0} \sim c_{s} / z_{m}$, with the coherent phase velocity $c_{s} \sim v_{m} \sqrt{U}$ [see Eq. (23)]. Large amplitude waves generated by the microwave instability can accelerate particles to velocities exceeding $c_{s}$ (see Figs. 11 and 12). Therefore one should include a safety factor of 2-3 for the barrier voltage, in order to ensure a penetration depth $z_{m}$ also for the tail particles. Or equivalently, in designing a barrier system for space charge dominated beams, especially close to the stability boundaries, the voltage should always be sufficiently high in order to confine the particle velocity $2-3 c_{s} \gg v_{m}$.

\section{B. Impedance budget considerations}

First, it is important to note that in the absence of a resistive impedance $(V=0)$ the perturbed sausage beam distribution is stable. Within our simulation model no tail formation or reconfiguration towards a more Gaussiantype distribution is observed. For finite $V$ and $U \lesssim 1$ an increased stability boundary relative to a equivalent coasting beam is observed in the simulation. In the space charge dominated regime $(U \geqslant 1)$ Eq. (27) or its approximation Eq. (28) can be used. The free parameter $\alpha_{e}$ depends, as observed in the simulations, on the ratio of the bunch length to the wavelength of the unstable mode. For known $\alpha_{e}$ and long bunches the maximum shunt impedance can be written in the simple form

$$
R_{s} \lesssim \frac{X_{\mathrm{sc}} L \alpha_{e}}{\pi l}
$$

with the circumference of the machine $L$, the space charge reactance $X_{\mathrm{sc}}$, and the bunch length $l$. The simulation shows that Eq. (30) might be used also to estimate the tolerable shunt impedance of barrier bucket rf cavities and its high harmonic impedance components.

In cooler storage rings it could be advantageous to cool in barrier buckets instead of using coasting beams. Because of the increased stability region in barrier buckets the heating due to coherent instabilities can be reduced or even totally suppressed. During cooling (reduction of
$\left.\Delta p / p_{0}\right)$ the $(U, V)$ parameters always move along a line that goes through the origin and the initial starting point. If the initial, "uncooled" beam parameters in the $(U, V)$ plane are within the stable area determined by Eq. (27) then also the cooled beam parameters will be stable.

\section{CONCLUSIONS}

The sausage beam model was introduced that extends the parabolic bunch model for usual rf buckets to barrier buckets. In the presence of space charge and for triangular barrier pulses the sausage beam model represents a strictly self-consistent, matched solution. The coherent stability limits of this distribution in the presence of a cavity resonator are studied using impedance scans performed with a Vlasov simulation code. The comparison between coasting and barrier bucket beams shows that space charge induced convection together with bunch end effects strongly widens the region of longitudinal stability, especially in the space charge dominated regime. A simple stability criterion was found in good agreement with the simulation results. The criterium can be very useful to estimate impedance budgets for space charge dominated beams in barrier bucket systems. The saturation of the instability in space charge dominated beams results in the formation of an extended longitudinal halo. For moderate space charge long-lived turbulent behavior is observed together with a stationary tentlike velocity distribution.

\section{ACKNOWLEDGMENTS}

The authors would like to thank H. Damerau, GSI for his very useful comments.

[1] E. Shaposhnikova, CERN Report No. SL-99-008/HRF, 1999.

[2] F. J. Sacherer, IEEE Trans. Nucl. Sci. 24, 1393 (1977).

[3] O. Boine-Frankenheim and I. Hofmann, Phys. Rev. ST Accel. Beams 3, 104202 (2000).

[4] E. P. Lee, in Proceedings of the Linear Accelerator Conference, Santa Fe, NM, 1981 (Report No. UCRL86452), p. 263.

[5] I. Hofmann, Z. Naturforsch 37A, 939 (1982).

[6] D. A. Callahan, A. B. Langdon, and A. Friedman, J. Appl. Phys. 81, 3398 (1997).

[7] J. M. Wang, D. X. Wang, H. Suk, and M. Reiser, Phys. Rev. Lett. 74, 3152 (1995).

[8] M. Fujieda et al., Phys. Rev. ST Accel. Beams 2, 122001 (1999).

[9] A. Blas, S. Hancock, M. Lindroos, and S. Koscielniak, in Proceedings of the EPAC 2000, Vienna, Austria (EPAC/ CERN, Geneva, 2000), p. 1528.

[10] "An International Accelerator Facility for Research with Ions and Antiprotons," Conceptual Design, GSI Report, 2002, http://www.gsi.de/GSI-Future/cdr/ 
[11] GSI Report No. 98-06, 1998, edited by I. Hofmann and G. Plass.

[12] T. Bohl, T. Linnecar, and E. Shaposhnikova, CERN Report No. CERN-SL-2000-020-HRF, 2000.

[13] S. Y. Lee and K. Y. Ng, Phys. Rev. E 55, 5992 (1997).

[14] K. Y. Ng, Phys. Rev. ST Accel. Beams 5, 061002 (2002).

[15] K. Takayama et al., Phys. Rev. Lett. 88, 144801 (2002).

[16] M. Weidemüller et al., AIP Conf. Proc. 457, 194 (1999).

[17] U. Eisenbarth et al., Nucl. Instrum. Methods Phys. Res., Sect. A 441, 209 (2000).

[18] J. J. Bisognano, in Proceedings of the 5th European Particle Accelerator Conference, Barcelona, Spain, 1996 (EPAC/CERN, Geneva, 1996), p. 328.

[19] A. Al-Khateeb, O. Boine-Frankenheim, I. Hofmann, and
G. Rumolo, Phys. Rev. E 63, 026503 (2001).

[20] D. Neuffer, Part. Accel. 11, 23 (1980).

[21] A. Hofmann and F. Pedersen, IEEE Trans. Nucl. Sci. 26, 3526 (1979).

[22] F. J. Sacherer, in Proceedings of the 1971 Particle Accelerator Conference, Chicago (IEEE, Piscataway, NJ, 1971), p. 1106.

[23] G. Franchetti, I. Hofmann, and G. Rumolo, Phys. Rev. ST Accel. Beams 3, 084201 (2000).

[24] M. Reiser, Theory and Design of Charged Particle Beams (Wiley, New York, 1994).

[25] P. Zenkevich, N. Mustafin, and O. Boine-Frankenheim, in Proceedings of the CERN ECLOUD'02 Workshop (CERN Yellow Report No. CERN-2002-001, 2002). 